IZA DP No. 8991

Benefit Reentitlement Conditions in Unemploment Insurance Schemes

Torben M. Andersen

Mark Strøm Kristoffersen

Michael Svarer

April 2015 


\title{
Benefit Reentitlement Conditions in Unemployment Insurance Schemes
}

\author{
Torben M. Andersen \\ Aarhus University \\ and IZA
}

Mark Strøm Kristoffersen

Danmarks Nationalbank

Michael Svarer

Aarhus University

and IZA

Discussion Paper No. 8991

April 2015

IZA

P.O. Box 7240
53072 Bonn
Germany

Phone: +49-228-3894-0

Fax: +49-228-3894-180

E-mail: iza@iza.org

Any opinions expressed here are those of the author(s) and not those of IZA. Research published in this series may include views on policy, but the institute itself takes no institutional policy positions. The IZA research network is committed to the IZA Guiding Principles of Research Integrity.

The Institute for the Study of Labor (IZA) in Bonn is a local and virtual international research center and a place of communication between science, politics and business. IZA is an independent nonprofit organization supported by Deutsche Post Foundation. The center is associated with the University of Bonn and offers a stimulating research environment through its international network, workshops and conferences, data service, project support, research visits and doctoral program. IZA engages in (i) original and internationally competitive research in all fields of labor economics, (ii) development of policy concepts, and (iii) dissemination of research results and concepts to the interested public.

IZA Discussion Papers often represent preliminary work and are circulated to encourage discussion. Citation of such a paper should account for its provisional character. A revised version may be available directly from the author. 
IZA Discussion Paper No. 8991

April 2015

\section{ABSTRACT}

\section{Benefit Reentitlement Conditions in Unemployment Insurance Schemes}

Unemployment insurance schemes include conditions on past employment history as part of the eligibility conditions. This aspect is often neglected in the literature which primarily focuses on benefit levels and benefit duration. In a search-matching framework we show that benefit duration and employment requirements are substitute instruments in affecting job search incentives and thus gross unemployment. We analyse the optimal design of the unemployment insurance system (benefit levels, duration and employment requirements) under a utilitarian social welfare function. Simulations show that a higher insurance motive captured by more risk aversion implies higher benefit generosity and more lax employment requirements but also shortened benefit duration.

JEL Classification: E32, H3, J65

Keywords: reentitlement effects, unemployment insurance, business cycle

Corresponding author:

Michael Svarer

Department of Economics and Business

Aarhus University

Fuglesangs Allé 4

DK - 8210 Aarhus V

Denmark

E-mail: michael@svarer.dk

\footnotetext{
* The viewpoints and conclusions stated are the responsibility of the authors, and do not necessarily reflect the views of Danmarks Nationalbank.
} 


\section{Introduction}

Unemployment benefit schemes are usually characterized in terms of benefit levels and duration. Eligibility conditions are equally important, in particular requirements to the previous employment record ${ }^{1}$ to initiate a benefit spell, e.g. a certain number of hours of work within a preceding period ${ }^{2}$. In the following we term this the "employment requirement". Benefit duration has to be seen relative to the employment requirement. If this requirement is lax, the maximum duration of a benefit spell matters less since it is easy to regain eligibility and vice versa. This suggest that employment requirements are an important aspect on par with benefit levels and duration in affecting incentives in the labour market.

While there is a voluminous literature on unemployment insurance (see e.g. Fredriksson \& Holmlund (2006) and Tatsiramos \& van Ours (2012) for surveys), it is only recently that an employment requirement has been explicitly included (see e.g. Hopenhayn \& Nicolini (2009), Ortega \& Rioux (2010), Pan \& Zhang (2012), and Zhang \& Faig (2012)). Hopenhayn \& Nicolini (2009) argue that if it is impossible to distinguish between quits and layoffs it is optimal to condition benefit eligibility on the employment history of unemployed. Ortega \& Rioux (2010) emphasize that an employment criterion can support job creation since unemployed who have exhausted their benefits are willing to accept lower wages to regain the right to unemployment benefits. However, none of those studies allow for endogenous search. We do so to shed light on the incentive role of employment requirements compared to those of benefit levels and duration.

Specifically we consider a search-matching framework with a two-tier benefit system (e.g. Mortensen (1977) and Fredriksson \& Holmlund (2001)) where unemployed receiving unemployment benefits transit to a lower benefit level (e.g. social assistance) when unemployment duration exceed some threshold. Our study is related to Ortega \& Rioux (2010). They, however, ignore the search effects of changing the parameters of the benefit system. Since the effects of unemployment insurance on job search incentives is crucial we allow for endogenous search. The main contribution of our paper is to compare the incentive effects of employment requirements to those of benefit levels and duration.

\footnotetext{
${ }^{1}$ Systems often have entry conditions specified in terms of eduvation and/or employment as well as employment requirements to initiate a new benefit spell.

${ }^{2}$ Venn (2012) gives a detailed description of unemployment benefit systems across OECD countries. She constructs quantitative indicators for the strictness of eligibility criteria for unemployment benefits across 36 OECD and/or EU members, showing large differences across fairly similar countries.
} 
We also consider the optimal design of the unemployment insurance scheme and how it depends on the need for insurance measure by risk aversion. We show that the reentitlement requirement can work as a substitute to the benefit duration in terms of generating incentives for job search.

The structure of the paper is as follows: In Section 2 we introduce a search-matching model with employment requirements for unemployment insurance eligibility. The effects of the employment requirement and other elements of the unemployment insurance scheme are analyzed in Section 3. The optimal UI scheme is considered in Section 4. Concluding remarks are given in Section 5.

\section{Benefit entitlement in a search-matching model}

Consider a search-matching model of the labor market where workers can be in one of four states: $i$ ) possessing a job and fulfilling the employment requirement for benefit eligibility in case of involuntary job-separation (state $E$ ), $i i$ ) possessing a job but not fulfilling the employment requirement and thus not being entitled to unemployment benefits $\left(b_{U}\right)$ but rather social benefits $\left(b_{K}<b_{U}\right)$ in case of involuntary job separation (state $N$ ), iii) being unemployed and entitled to unemployment benefits (state $U$ ), UIB-unemployed, and $i v$ ) being unemployed and not entitled to unemployment benefits but social assistance (state $K)$, SA-unemployed. There is a continuum of workers with mass one. All workers are assumed to own an equal share of the firms, and therefore firm profits are distributed among workers, which secures that our model can be given a general equilibrium interpretation. All employed may lose their job by the exogenous separation rate $p_{U, E}$. Employed not entitled to unemployment benefits gain eligibility at the rate $p_{E, N}$, and thus the expected length of the employment period to regain UIB eligibility is $1 / p_{E, N}$. Unemployed eligible for benefits search for jobs at the rate $s_{U}$ and find a job at the rate $\alpha s_{U}$, where $\alpha$ is the job-finding rate (see below). Unemployed eligible for benefits lose eligibility at the rate $^{3} p_{K, U}$, and thus the expected potential duration of benefit receipt is $1 / p_{K, U}$. Finally, unemployed non-eligible for benefits search at the rate $s_{K}$ and thus find a job at the rate $\alpha s_{K}$. Note that there are only involuntary job-separations in the model.

The instantaneous utility to an employed is

$$
h\left(I_{i}, 1-l\right) ; i=E, N
$$

\footnotetext{
${ }^{3}$ That is, we follow Fredriksson \& Holmlund (2001) who show that a fixed time duration can be approximated by a system in which there is a stochastic transition from one benefit level to another.
} 
where $I_{i}$ is net income given as labor income after $\operatorname{tax}^{4} w_{i}[1-\tau]$ and its share of profits $\Pi$. Time endowment is normalized to unity and working hours are $l$ (exogenous).

The instantaneous utility for unemployed is ${ }^{5}$

$$
g\left(I_{j}, 1-s_{j}\right) ; j=U, K
$$

where income is the sum of the transfer $\left(b_{U}\right.$ or $\left.b_{K}\right)$ and the profit share $\Pi$, and $s_{j}$ is the amount of time spent searching for a job. The functions $h$ and $g$ have the standard properties.

The value functions associated with the four possible labor market states are

$$
\begin{aligned}
\rho V_{E} & =h\left(w_{E}[1-\tau]+\Pi, 1-l\right)+p_{U, E}\left[V_{U}-V_{E}\right] \\
\rho V_{N} & =h\left(w_{N}[1-\tau]+\Pi, 1-l\right)+p_{U, E}\left[V_{K}-V_{N}\right]+p_{E, N}\left[V_{E}-V_{N}\right] \\
\rho V_{U} & =g\left(b_{U}+\Pi, 1-s_{U}\right)+\alpha s_{U}\left[V_{E}-V_{U}\right]+p_{K, U}\left[V_{K}-V_{U}\right] \\
\rho V_{K} & =g\left(b_{K}+\Pi, 1-s_{K}\right)+\alpha s_{K}\left[V_{N}-V_{K}\right]
\end{aligned}
$$

where $\rho$ is the discount rate, $b_{U}>b_{K}$ (see below).

We want to focus on differences or asymmetries arising solely from the design of the unemployment insurance system, and hence we essentially assume that all workers are identical except for their labor market history and thus possibly their benefit entitlement. In this spirit we have assumed that $p_{K, N}=p_{U, E}$; i.e., the job separation rate is the same for eligible and non-eligible workers, and they have the same working hours (exogenous). ${ }^{6}$ Note also that the participation constraints: $V_{E} \geq V_{U}, V_{N} \geq V_{K}, V_{E} \geq V_{N}, V_{U} \geq V_{K}$ are assumed fulfilled in the following.

In the following this short-hand notation will be used

$$
\begin{aligned}
h_{i}(\cdot) & \equiv h\left(w_{i}[1-\tau]+\Pi, 1-l\right) \text { for } i=E, N \\
g_{j}(\cdot) & \equiv g\left(b_{j}+\Pi, 1-s_{j}\right) \text { for } j=U, K .
\end{aligned}
$$

\footnotetext{
${ }^{4}$ Labour income is taxed while profits is not. Note that the tax is financing expenditures on unemployment benefits and social assistance. hence, this formulation captures actual modes of financing via social contributions or taxes.

${ }^{5}$ For notational reasons, we allow the instantaneous utility function of the unemployed to differ from that of the employed. However, our results will not hinge on this asymmetry, and in the numerical illustrations $h(\cdot)=g(\cdot)$.

${ }^{6}$ We impose this symmetry to focus on the question whether re-entitlement conditions can be motivated as a means to improve the trade-off between incentives and insurance in the social safety net.
} 
The individual takes all variables except the search level to be beyond its own control (i.e., to be unaffected by its decisions), and thus the optimal search level for the two types of unemployed is determined by (note that standard assumptions on $g$ ensure that the second order condition is fulfilled)

$$
\begin{aligned}
& \frac{\partial g_{U}(\cdot)}{\partial\left(1-s_{U}\right)}=\alpha\left[V_{E}-V_{U}\right] \\
& \frac{\partial g_{K}(\cdot)}{\partial\left(1-s_{K}\right)}=\alpha\left[V_{N}-V_{K}\right] .
\end{aligned}
$$

Denoting the share of the population receiving unemployment benefits and social assistance by $u$ and $k$, respectively, we have that total search is given as

$$
s \equiv s_{U} u+s_{K} k
$$

A standard constant returns to scale matching function defined over total search and vacancies $(v)$ is assumed

$$
m(s, v) \text {. }
$$

The function $m$ has the usual properties. It follows that the job-finding rate is given by

$$
\alpha=\frac{m(s, v)}{s}=m(1, \theta)
$$

where $\theta \equiv \frac{v}{s}$ is market tightness, and hence $\alpha=\alpha(\theta), \alpha^{\prime}(\theta)>0$. The job filling rate is

$$
q=\frac{m(s, v)}{v}=m\left(\theta^{-1}, 1\right)
$$

and thus $q=q(\theta), q^{\prime}(\theta)<0$.

Firms post vacancies to find vacant workers, and in the hiring process they are not able to distinguish workers by their eligibilities in the unemployment insurance scheme. Thus, the value of a vacancy $\left(J_{V}\right)$ is expressed in terms of the expected value of a filled job $\left(J_{E X P}\right)$; i.e.,

$$
\rho J_{V}=-\kappa+q\left[J_{E X P}-J_{V}\right]
$$

where $\kappa$ is the flow vacancy cost. The free-entry-condition, $J_{V}=0$, then implies

$$
J_{E X P}=\frac{\kappa}{q}
$$

where $J_{E X P}$ is the expected value of a filled job; that is

$$
J_{E X P} \equiv \frac{\alpha s_{U} u J_{E}+\alpha s_{K} k J_{N}}{\alpha s_{U} u+\alpha s_{K} k}=\frac{s_{U} u J_{E}+s_{K} k J_{N}}{s_{U} u+s_{K} k} .
$$


After a firm and a worker are matched, the firm knows whether the candidate is entitled to UIB or SA, and therefore the wage depends on the worker's UIB eligibility. The value of a job filled with a UIB eligible worker is ${ }^{7}$

$$
\rho J_{E}=y-w_{E}+p_{U, E}\left[J_{V}-J_{E}\right]
$$

and the value of a job filled with a worker non-eligible for unemployment benefits is

$$
\rho J_{N}=y-w_{N}+p_{E, N}\left[J_{E}-J_{N}\right]+p_{U, E}\left[J_{V}-J_{N}\right] .
$$

Wages are determined through Nash bargaining; i.e.,

$$
\begin{aligned}
& w_{E}=\arg \max _{w_{E}}\left(V_{E}-V_{U}\right)^{\beta}\left(J_{E}-J_{V}\right)^{1-\beta} \\
& w_{N}=\arg \max _{w_{N}}\left(V_{N}-V_{K}\right)^{\beta}\left(J_{N}-J_{V}\right)^{1-\beta}
\end{aligned}
$$

with the associated first-order conditions (second order conditions assumed to be fulfilled)

$$
\begin{aligned}
& \beta \frac{\frac{\partial V_{E}}{\partial w_{E}}}{V_{E}-V_{U}}+(1-\beta) \frac{\frac{\partial J_{E}}{\partial w_{E}}}{J_{E}}=0 \\
& \beta \frac{\frac{\partial V_{N}}{\partial w_{N}}}{V_{N}-V_{K}}+(1-\beta) \frac{\frac{\partial J_{N}}{\partial w_{N}}}{J_{N}}=0 .
\end{aligned}
$$

Profits are given as

$$
\Pi=\left[y-w_{E}\right] e+\left[y-w_{N}\right] n-v \kappa
$$

where $e$ and $n$ denote the number of eligible and non-eligible workers, respectively. The inflow and outflow equations read (where $e=1-u-k-n$ )

$$
\begin{gathered}
U: \quad c p_{U, E}=\alpha s_{U} u+p_{K, U} u \\
K \quad: \quad n p_{U, E}+p_{K, U} u=\alpha s_{K} k \\
N \quad: \quad \alpha s_{K} k=p_{U, E} n+p_{E, N} n
\end{gathered}
$$

for unemployment, social assistance and non-eligible jobs, respectively.

\footnotetext{
${ }^{7}$ Note that workers gaining eligibility for $b_{u}$ experience an immediate change in the wage since the worker and the firm are implicitly assumed to renegotiate the wage promptly. This assumption may be empirically questionable, but we make it for tractability reasons.
} 
For later reference note that the fraction of the population in the various labor market states can be written (recall that $1=e+n+u+k$ )

$$
\begin{aligned}
e & =e\left(\alpha, s_{U}, s_{K}, p_{E, N}, p_{K, U}, p_{U, E}\right) \\
n & =n\left(\alpha, s_{U}, s_{K}, p_{E, N}, p_{K, U}, p_{U, E}\right) \\
u & =u\left(\alpha, s_{U}, s_{K}, p_{E, N}, p_{K, U}, p_{U, E}\right) \\
k & =k\left(\alpha, s_{U}, s_{K}, p_{E, N}, p_{K, U}, p_{U, E}\right) .
\end{aligned}
$$

Finally, the public budget constraint reads

$$
\tau\left(w_{E} e+w_{N} n\right)=b_{U} u+b_{K} k
$$

To sum up, the unemployment insurance scheme is characterized by two benefit levels $\left(b_{U}, b_{K}\right)$, the transition out of $b_{U}\left(p_{K, U}\right)$, and the entry into $b_{U}$ eligibility $\left(p_{E, N}\right)$ capturing reentitlement requirements. Recall that expected benefit duration is $1 / p_{K, U}$ and the employment requirement period $1 / p_{E, N}$.

In summary, the equilibrium to the model is characterized by unemployed choosing search effort according to (1) and (2), firms creating vacancies according to (3), wages determined by (4) and (5), the tax rate determined from (9) and the flow equations (6), (7), and (8). It can be shown ${ }^{8}$ that the resource balance condition (or goods-market equilibrium condition) is fulfilled; i.e., aggregate output (net of vacancy costs) equals aggregate consumption.

\subsection{Calibration}

In the next section we derive some partial, analytical results on the effects on job search and gross unemployment from changing benefit duration and the entitlement requirement. In general, however, there a few clear-cut analytical results and therefore we use a numerical illustration when necessary.

As explained further below we use the baseline calibration of Ortega \& Rioux (2010) as well as their functional forms (where possible). The time period is a month. The job separation rate $\left(p_{U, E}\right)$ is 0.00977 . Productivity ${ }^{9}(y)$ is 1.517 in the benchmark, and

\footnotetext{
${ }^{8}$ For proof see Appendix available upon request from the authors.

${ }^{9}$ Productivity and the vacancy cost are rescaled (by 1/1000) compared to Ortega \& Rioux to ensure that the choice of search intensities delivers interior solutions. This rescaling has no qualitative effects.
} 
the flow cost of vacancies $(\kappa)$ is 0.56181 . The discount rate $(\rho)$ is 0.01 . We assume the following functional forms for instantaneous utilities

$$
\begin{gathered}
h\left(I_{i}, 1-l\right)=\frac{1}{1-\gamma} I_{i}^{1-\gamma}+\log (1-l) \quad ; i=E, N \\
g\left(I_{i}, 1-s_{i}\right)=\frac{1}{1-\gamma} I_{i}^{1-\gamma}+\log \left(1-s_{i}\right) ; i=U, K
\end{gathered}
$$

with $\gamma=1.5$, and workers are assumed to spend $40 \%$ of their time at work; i.e., $l=0.4$.

The matching function is Cobb-Douglas

$$
m(s, v)=A s^{\varepsilon} v^{1-\varepsilon}
$$

with $A=0.05$ and $\varepsilon=0.5$. To avoid introducing arbitrary inefficiencies we set $\beta=\varepsilon=$ 0.5 .

\section{Search and properties of the UIB system}

We start out by clarifying the role of benefit duration $\left(p_{K, U}\right)$ and benefit entitlement $\left(p_{E, N}\right)$ for given benefit levels $\left(b_{U}, b_{K}\right)$ for labor market performance; that is, we consider the implication for a given macroeconomic environment, i.e., wages $\left(w_{E}, w_{N}\right)$, taxes $(\tau)$, and job-finding rate $(\alpha)$. This clarifies the direct incentive/search effects of the two instruments as part of the social safety net. The complexity of the overall model makes it impossible to arrive at any analytical results for the general equilibrium effects, but the partial approach gives some important insights on the role of the various elements in the unemployment insurance system, and also for the interpretation of the numerical results presented below.

It can be shown ${ }^{10}$ that for a given macroeconomic environment

$$
\begin{aligned}
& \frac{d s_{U}}{d p_{K, U}}>0 ; \frac{d s_{K}}{d p_{K, U}}<0 \\
& \frac{d s_{U}}{d p_{E, N}}<0 ; \frac{d s_{K}}{d p_{E, N}}>0
\end{aligned}
$$

i.e., shorter benefit duration (higher $p_{K, U}$ ) implies that the UIB-unemployed search more to enhance the chance of finding a job in light of the more dire consequences if this is not successful, and for the SA-unemployed search becomes less attractive since the value of finding a job leading to UIB entitlement is now lower. A more lax employment

\footnotetext{
${ }^{10}$ For proof of results in this section see Appendix available upon request from the authors.
} 
requirement (higher $p_{E, N}$ ) makes the non-entitled unemployed search more since the value of a job is now higher since it more easily leads to UIB-entitlement. Oppositely the eligible unemployed search less since it is less critical to lose entitlement as it can more easily be regained.

Note that the two instruments, benefit duration and entitlement conditions, have different implications for the two types of unemployed. Considering the marginal rate of substitution between the two instruments leaving the utility gain from finding a job unchanged for entitled and non-entitled, respectively, we have (for proof and notation see Appendix)

$$
\begin{aligned}
& \frac{d p_{K, U}}{d p_{E, N}} \mid\left[V_{E}-V_{U}\right]=\text { constant }=\frac{\frac{p_{K, U}}{\rho} \frac{\alpha s_{K}}{B_{1}}\left[\frac{1}{\rho} B_{2}-\left[V_{N}-V_{K}\right]\left[1+\frac{1}{\rho} \alpha s_{K}\right]\right]}{\left[\frac{1}{\rho} A_{2}-\left[V_{E}-V_{U}\right]\left(1+\frac{p_{U, E}}{\rho}\right)\right]}>0 \\
& \frac{d p_{E, N}}{d p_{K, U}} \mid\left[V_{N}-V_{K}\right]=\mathrm{constant}=\frac{\frac{p_{E, N}}{\rho} \frac{p_{U, E}}{A_{1}}\left[\frac{1}{\rho} A_{2}-\left[V_{E}-V_{U}\right]\left(1+\frac{p_{U, E}}{\rho}\right)\right]}{\left[\frac{1}{\rho} B_{2}-\left[V_{N}-V_{K}\right]\left[1+\frac{1}{\rho} \alpha s_{K}\right]\right]}>0 .
\end{aligned}
$$

Note that search levels are unchanged for utility gains being constant $\left(V_{E}-V_{U}=\right.$ constant, and $V_{N}-V_{K}=$ constant). The above thus gives the combinations of $p_{K, U}$ and $p_{E, N}$, leaving search $\left(s_{U}\right.$ and $s_{K}$ respectively) unchanged. Both types' iso-search curves are upward sloping in the $\left(p_{K, U}, p_{E, N}\right)$-spaces; that is, a higher rate $\left(p_{E, N}\right)$ at which nonentitled become entitled to UIB (easier UIB entitlement) has to be accompanied with a higher rate $\left(p_{N, U}\right)$ at which UIB-unemployed lose their UIB entitlement (shorter benefit duration) to leave search unchanged, i.e., the two instruments are substitutes. This applies for both types of unemployed, but the marginal rates of substitution are different, which suggests that it may be desirable to let the UI scheme feature both elements (see below). Intuitively, using two instruments seems to dominate using only one since there are two search levels which can be affected, and the two instruments are not perfect substitutes, cf. the difference in the marginal rates of substitution.

Finally, note that (for proof see Appendix) an increase in $p_{K, U}$ leads to a decrease in $V_{E}$ and a decrease in $V_{K}$, while there is an ambiguous effect on $V_{N}$ and $V_{U}$. An increase in $p_{E, N}$ leads to an increase in $V_{E}$ and an increase in $V_{K}$, while there is an ambiguous effect on $V_{N}$ and $V_{U}$. 


\subsection{Labor market outcomes}

Consider next the role of benefit duration and employment eligibility conditions for labour market performance assessed in terms of the gross level of unemployment $(u+k)$, i.e. recipients of unemployment benefits or social assistance.

From the flow equations we have

$$
[e+n] p_{U, E}=\alpha s_{U} u+\alpha s_{K} k=\alpha s
$$

i.e., for a given job-separation rate $p_{U, E}$ and job-finding rate $\alpha$, total employment $(e+n)$ is monotonously increasing in aggregate search. Since $1=e+n+u+k$ it follows that $u+k$ is monotonously decreasing in $s$.

We show in the Appendix that the gross unemployment rate $(u+k=1-(e+n))$ can be written in implicit form as

$$
u+k=F\left(s_{U}\left(p_{K, U}, p_{E, N}\right), s_{K}\left(p_{K, U}, p_{E, N}\right), p_{K, U}, p_{E, N}\right) .
$$

It can be shown that (see Appendix)

$$
\begin{aligned}
\frac{\partial F(\cdot)}{\partial s_{U}} & <0 ; \frac{\partial F(\cdot)}{\partial s_{K}}<0 \\
\operatorname{sign}\left(\frac{\partial F(\cdot)}{\partial p_{K, U}}\right) & =\operatorname{sign}\left(s_{U}-s_{K}\right) \\
\operatorname{sign}\left(\frac{\partial F(\cdot)}{\partial p_{E, N}}\right) & =-\operatorname{sign}\left(\frac{\partial F(\cdot)}{\partial p_{K, U}}\right) .
\end{aligned}
$$

It is an implication that for given search there is always a trade-off between benefit duration and benefit entitlement in achieving a given level of gross unemployment; i.e., if benefit eligibility can be acquired more easily (higher $p_{E, N}$ ), then benefit duration can be reduced (higher $p_{K, U}$ ).

In general search responses imply that the slope of the iso-employment locus cannot be unambiguously signed. Therefore, we turn to a numerical illustration and Figure 1 shows iso-gross unemployment curves in the $\left(p_{E, N}, p_{K, U}\right)$-plane for the full model where e.g. wages and labour tightness are no longer fixed (see above for the numerical details). We see that the iso-gross unemployment curves are positively sloped, which reveals a trade-off between easier reentitlement and shorter UIB duration in sustaining a certain level of $u+k$, i.e. the partial results found above are also found for the general equilbrrium outcome. Furthermore, gross unemployment increases when we move to the South-East, i.e., longer UIB duration and/or more lax employment requirements. This is explained by SA-unemployed searching more intensively than UIB-unemployed $\left(s_{K}>s_{U}\right)$. 
Figure 1: Iso-gross unemployment Curves, trade-off Between UIB

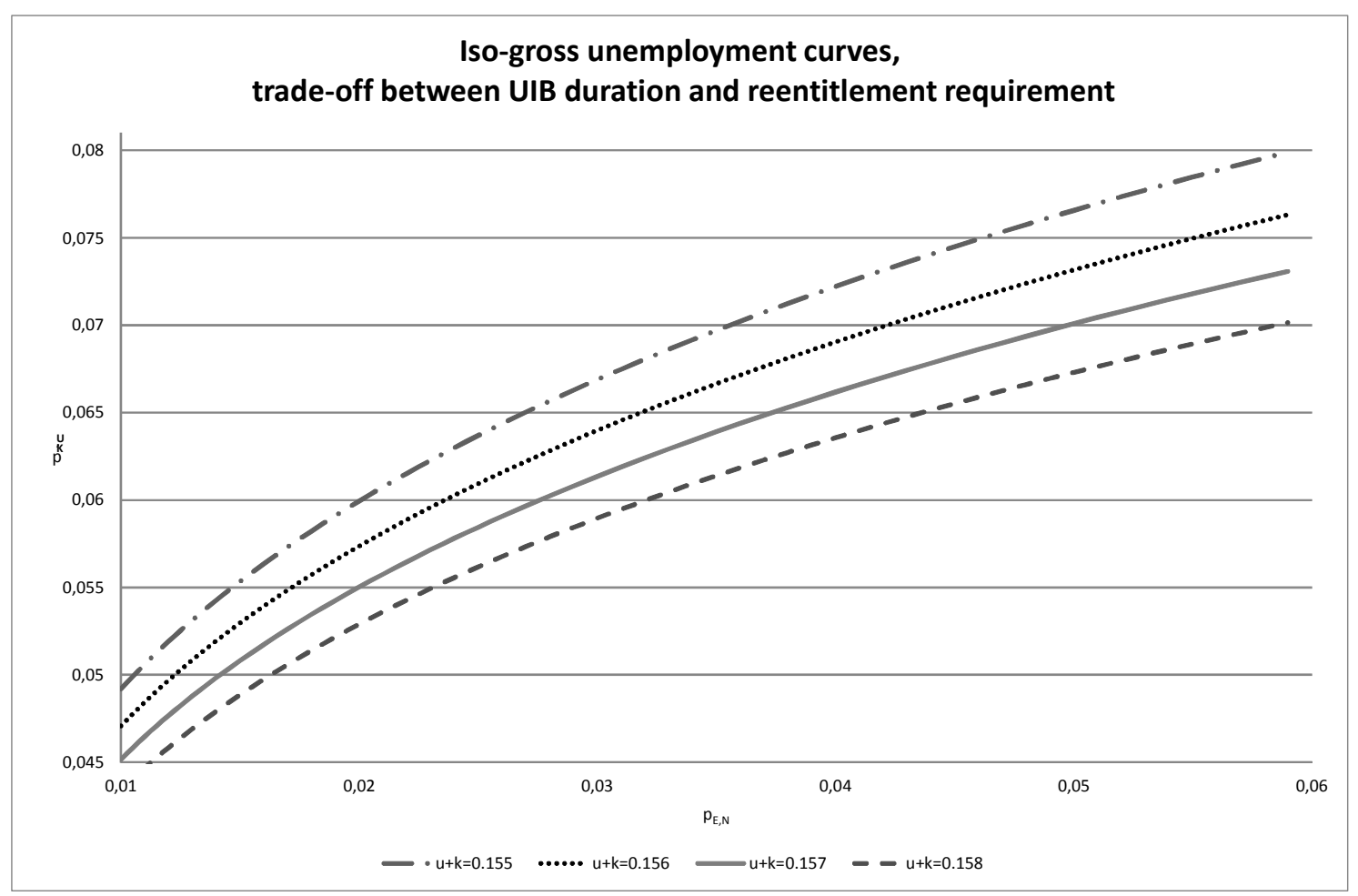

\section{Optimal social safety net}

In the following we consider the optimal design of the social safety net assuming a utilitarian social welfare function. The social welfare function can be written ${ }^{11}$

$$
\begin{aligned}
\Omega & =e \rho V_{E}+n \rho V_{N}+u \rho V_{U}+k \rho V_{K} \\
& =e h_{E}(\cdot)+n h_{N}(\cdot)+u g_{U}(\cdot)+k g_{K}(\cdot) .
\end{aligned}
$$

The social welfare function $\Omega$ gives the sum of utility generated in the economy under a given policy package $\left(b_{U}, b_{K}, p_{K, U}, p_{E, N}\right)$.

As a prelude it is useful to consider some special cases. The standard case considered in the literature assumes that employment automatically gives entitlement to unemployment

\footnotetext{
${ }^{11}$ For proof see Appendix available upon request from the authors.
} 
benefits in the case of layoffs corresponding to $p_{E, N} \rightarrow \infty$, in which case $n \rightarrow 0$. A simple one-tier benefit scheme in this case arises if $p_{K, U} \rightarrow 0$ (infinite benefit duration) implying $k \rightarrow 0$. Note also that $p_{E, N} \rightarrow 0$ implies that it is not possible to transit to a job providing entitlement to unemployment benefits in the case of lay-off, and hence ${ }^{12} u \rightarrow 0$ and $e \rightarrow 0$; i.e., this case corresponds to a one-tier benefit scheme where the only two states are $N$ and $K$.

There are two pertaining questions on the optimal design of the unemployment insurance scheme:

i) Is it optimal to have two tiers, i.e., to have a finite duration of unemployment benefits after which unemployed transit to a lower social assistance, corresponding to $p_{K, U}>0$ ?

ii) Is it optimal to include an employment condition as part of the eligibility conditions in the benefit scheme, i.e., to have $0<p_{E, N}<\infty$ ?

A sufficient ${ }^{13}$ condition for the optimal UI scheme to have two tiers is

$$
\lim _{p_{K, U} \rightarrow 0} \frac{\partial \Omega}{\partial p_{K, U}}>0
$$

whereas a sufficient condition for the optimality of a reentitlement condition is

$$
\lim _{p_{E, N} \rightarrow \infty} \frac{\partial \Omega}{\partial p_{E, N}}<0
$$

conditional on two tiers being optimal. The overall complexity of the model prevents us from analytically deriving clear answers to the two key questions, and therefore we resort to a numerical illustration below.

The reasoning above suggests that the design of the optimal unemployment insurance scheme is by no means trivial ${ }^{14}$. To proceed we adopt the calibration of Ortega \& Rioux (2010), see details above, in which case the optimal social safety net exhibits two tiers and non-automatic UIB-entitlement. Clearly, the results are dependent on the chosen functional forms and parameter values.

\footnotetext{
${ }^{12}$ Note that the flow equations imply $n p_{E, N}=p_{K, U} u$.

${ }^{13}$ Rigorously speaking, the sufficient condition should also include $\lim _{p_{K, U} \rightarrow \infty} \frac{\partial \Omega}{\partial p_{K, U}}<0$. However, in the limit there is only notational difference between a one-tier scheme with only SA-unemployed and a one-tier scheme with only UIB-unemployed.

${ }^{14}$ Numerical solutions of the model underpin this ambiguity since a two-tier benefit scheme with nonautomatic entitlement for UIB is by no means a universal solution to the problem of choosing the optimal structure of the social safety net.
} 


\subsection{Optimal UI scheme}

The numerical solution has the optimal UI scheme to include two tiers and non-automatic entitlement for UIB, i.e., $b_{U}>b_{K}>0,0<p_{K, U}<\infty$, and $0<p_{E, N}<\infty$. In particular, welfare defined in (10) is maximized with $b_{U}=0.587, b_{K}=0.207, p_{K, U}=0.057$, and $p_{E, N}=0.023$. Gross unemployment $(u+k)$ is approximately $16 \%$, the replacement rate for UIB eligible workers $\left(R R_{U} \equiv \frac{b_{U}}{w_{E}(1-\tau)}\right)$ is $58 \%$, while it is only $23 \%$ for non-eligible workers $\left(R R_{K} \equiv \frac{b_{K}}{w_{N}(1-\tau)}\right)$.

The interesting question is the role played by the four dimensions of the unemployment insurance scheme $\left(b_{U}, b_{K}, p_{K, U}, p_{E, N}\right)$ in the optimal policy. Underlying this is a question of insurance vs. incentives. The unemployment insurance scheme is there to protect against income losses in case of unemployment, and at the same time it should be designed so as to support job search incentives. Properties of this trade-off can be explored by varying the parameter $(\gamma)$ capturing the relative risk aversion of individuals and thus the need for insurance. As is well-known the utilitarian planner redistributes depending on marginal utilities of consumption. By increasing $\gamma$ and thus making marginal utilities more sensitive to income/consumption implies, other things being equal, that there is a stronger motive to redistribute. This calls for higher levels of benefits $\left(b_{U}\right)$ and social assistance $\left(b_{K}\right)$. This raises the classical issue of efficiency vs equity. Can and should the disincentive effects of these increases be countered by changes in benefit duration and the employment requirement?

In Figure 2 we show the response of the four dimensions of the unemployment insurance system to an increase in the risk aversion. As expected both the unemployment benefit and the social assistance are increasing in risk aversion for the reasons discussed above. However, benefit duration is monotonously decreasing in risk aversion $\left(p_{K, U}\right.$ is increasing). For the reentitlement requirement we find a non-monotone path. Starting from a low level, an increase in risk aversion first causes renetitlement conditions to be tightened ( $p_{E, N}$ decreases) and then at higher levels to be loosened ( $p_{E, N}$ increases). For risk above a critical level, an increase in risk aversion leads to more generosity along three dimensions; higher benefits, higher social assistance, and easier reentitlement conditions, but a tigthening in the form of shorter benefit duration. The subsitution between benefit duration and entitlement thus carries over to optimal policies. This suggests that benefit duration plays a more important role than the employment conditions in maintaining the incentive structure when more insurance is provided since agents are more risk averse. 


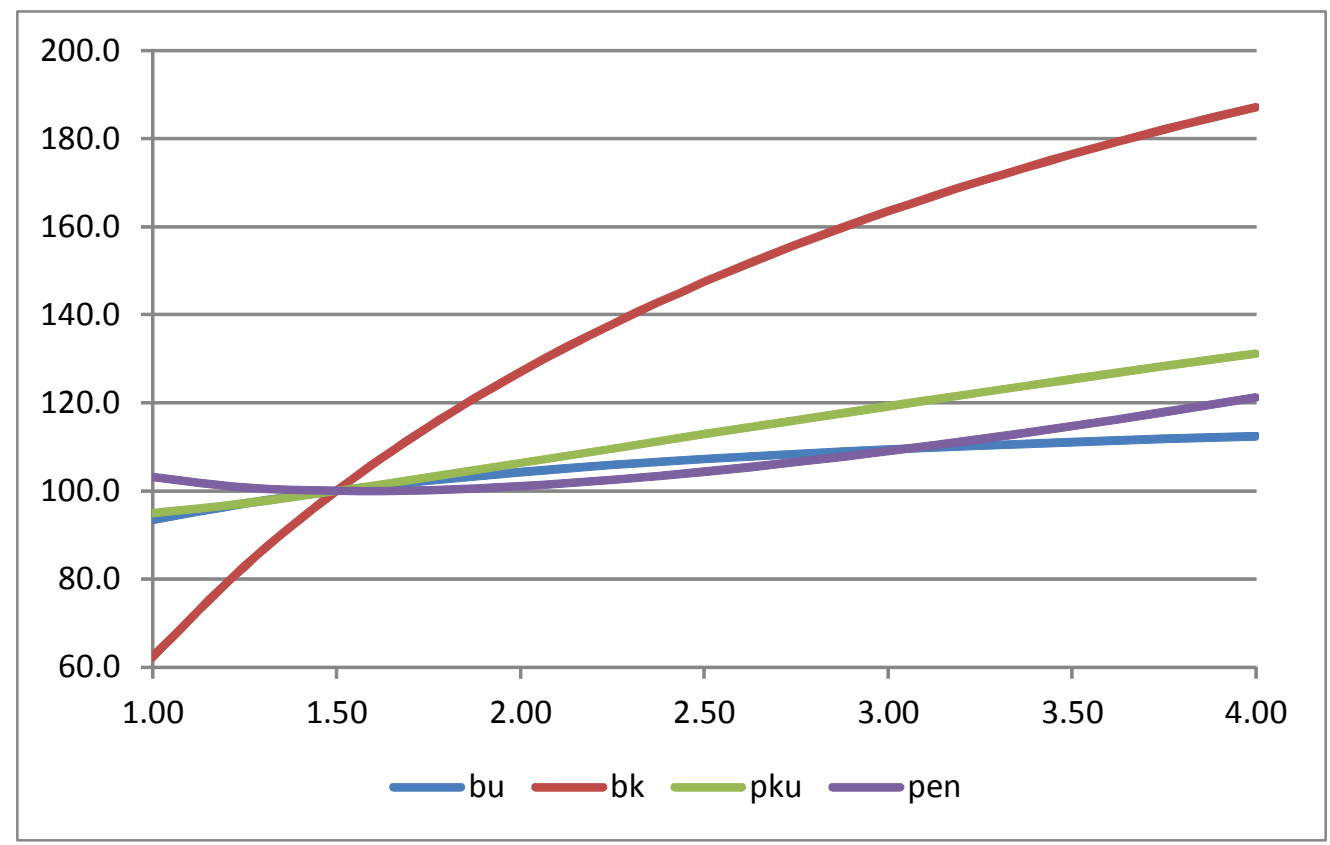

Note: variables are indexed relative to the benchmark case, index $=100$.

To shed more light on the role benefit duration and entitlement conditions play in balancing incentive and insurance effects, we show the optimal levels of unemployment benefits and social assistance for given levels of benefit duration and entitlement conditions ( i.e. constant levels of $p_{K, U}$ and $p_{E, N}$ ). The key findings are reported in Figure 3. When duration and entitlement conditions cannot be adjusted we find that there is a critical level of risk aversion, below which both types transfers are higher and above which lower than when all four dimensions can be adjusted to the degree of risk aversion. Above this critical level we thus find that more restricted adjustment possibilities lead to less generous transfers, which indicate that the incentive problems are larger in the restricted than in the unrestricted case. 
Figure 3: Optimal unemployment Benefits and Social assistance: All INSTRUMENTS CHOSEN OPTIMALLY VS ONLY BENEFIT LEVELS CHOSEN OPTIMALLY

Optimal UI level

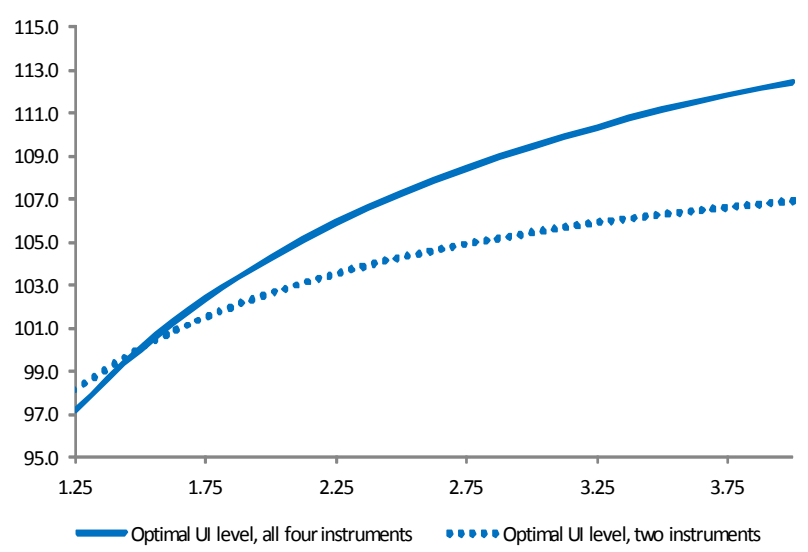

Optimal SA level

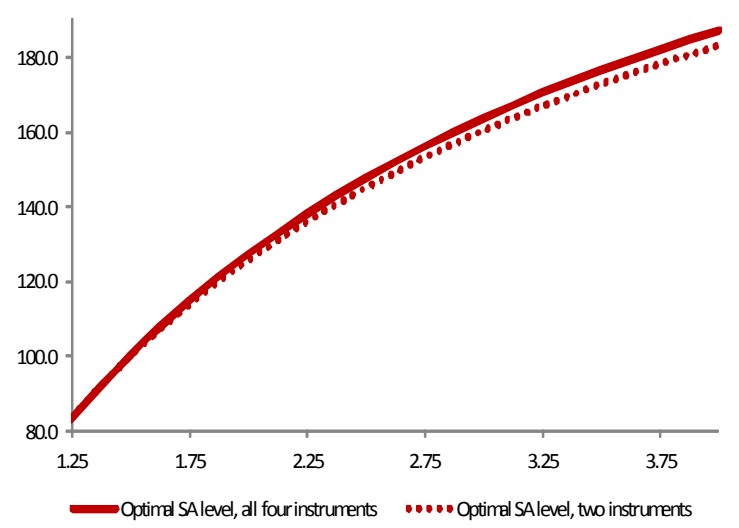

\section{Concluding remarks}

In this paper we have considered the role of unemployment benefit reentitlement conditions when designing the optimal unemployment insurance (UI) scheme in a searchmatching framework. We have shown that a reentitlement requirement can work as a substitute to the benefit duration. We have characterized the optimal design of the unemployment insurance scheme and considered how it depends on the need for insurance captured by risk aversion of households. Interestingly we find that although more risk aversion in general calls for higher benefit levels it also implies shorter benefit duration. The optimal reentitlement condition is non-monotone in risk aversion. Increasing risk aversion from low levels implies longer employment to requalifiy for UI benefits, whereas increasing risk aversion at higher levels implies shorter employment to requalify for UI benefits. These findings suggest that benefit duration plays a larger role in maintaining incentives when more insurance is demanded. 


\section{References}

[1] Fredriksson, P. \& B. Holmlund (2001), Optimal Unemployment Insurance in Search Equilibrium, Journal of Labor Economics, 19(2), 370-399.

[2] Fredriksson, P. \& B. Holmlund (2006), Incentives in Unemployment Insurance: A Review of the Recent Literature, Journal of Economic Surveys, 20(3), 357-386.

[3] Hopenhayn, H. \& J. P. Nicolini (2009), Optimal Unemployment Insurance and Employment History, Review of Economic Studies, 76, 1049-1070.

[4] Mortensen, D. T. (1977), Unemployment Insurance and Job Search Decisions, Industrial and Labor Relations Review, 30, 505-517.

[5] Ortega, J. \& L. Rioux (2010), On the Extent of Re-Entitlement Effects in Unemployment Compensation, Labour Economics, 17, 368-382.

[6] Pan, J. \& M. Zhang (2012), Optimal Unemployment Insurance with Endogenous UI Eligibility, manuscript, Fudan University.

[7] Tatsiramos, K. \& J. C. van Ours (2012), Labor Market Effects of Unemployment Insurance Design, IZA Discussion Paper No. 6950.

[8] Venn, D. (2012), Eligibility Criteria for Unemployment Benefits: Quantitative Indicators for OECD and EU Countries, OECD Social, Employment and Migration Working Papers, 131.

[9] Zhang, M. \& M. Faig (2012), Labor Market Cycles, Unemployment Insurance Eligibility, and Moral Hazard, Review of Economic Dynamics, 15, 41-56. 


\section{Appendix: Search and the properties of the unem- ployment insurance scheme}

The first order conditions determining search can conveniently be written

$$
\begin{gathered}
\Lambda_{s}^{U}\left(s_{U}, b_{U}, \tau, \alpha, V_{E}-V_{U}\right) \equiv-\frac{\partial g\left(b_{U}+\Pi, 1-s_{U}\right)}{\partial s_{U}}+\alpha\left[V_{E}-V_{U}\right]=0 \\
\Lambda_{s}^{K}\left(s_{K}, b_{K}, \tau, \alpha, V_{N}-V_{K}\right) \equiv-\frac{\partial g\left(b_{K}+\Pi, 1-s_{K}\right)}{\partial s_{K}}+\alpha\left[V_{N}-V_{K}\right]=0
\end{gathered}
$$

and the associated second order conditions read (sub-indices indicate derivatives wrt. to the variable stated)

$$
\begin{aligned}
& \Lambda_{s s}^{U}(\cdot)<0 \\
& \Lambda_{s s}^{K}(\cdot)<0 .
\end{aligned}
$$

In the following the benefits levels $\left(b_{U}, b_{K}\right)$ are given, as are all "macro variables" $\left(\tau, \alpha, w_{U}, w_{N}\right)$, and we are interested in the role of $p_{K, U}$ and $p_{E, N}$. Totally differentiating we find

$$
\Lambda_{s s}^{U} d s_{U}+\Lambda_{s z}^{U} d z=0 \text { for } z=p_{K, U}, p_{E, N} .
$$

It follows that

$$
\frac{d s_{U}}{d z}=-\frac{\Lambda_{s z}^{U}}{\Lambda_{s s}^{U}}
$$

and hence

$$
\operatorname{Sign}\left[\frac{d s_{U}}{d z}\right]=\operatorname{Sign}\left[\Lambda_{s z}^{U}\right]
$$

where

$$
\Lambda_{s z}^{U}=\alpha \frac{d\left[V_{E}-V_{U}\right]}{d z}, \text { with } z=p_{K, U}, p_{E, N} .
$$

Similar expressions apply for $s_{K}$.

Hence, to clarify how $\left(p_{K, U}, p_{E, N}\right)$ affect search for U- and K-types we need to know how $V_{E}-V_{U}$ and $V_{N}-V_{K}$ are affected. Defining the short-hands

$$
\begin{aligned}
h_{E}(\cdot) & \equiv h\left(w_{E}[1-\tau]+\Pi, 1-l\right) \\
h_{N}(\cdot) & \equiv h\left(w_{N}[1-\tau]+\Pi, 1-l\right) \\
g_{U}(\cdot) & \equiv g\left(b_{U}+\Pi, 1-s_{U}\right) \\
g_{K}(\cdot) & \equiv g\left(b_{K}+\Pi, 1-s_{K}\right)
\end{aligned}
$$

and using the value functions, we have

$$
\begin{aligned}
\rho\left[V_{E}-V_{U}\right] & =h_{E}(\cdot)-g_{U}(\cdot)+p_{U, E}\left[V_{U}-V_{E}\right]-\alpha s_{U}\left[V_{E}-V_{U}\right]-p_{K, U}\left[V_{K}-V_{U}\right] \\
\rho\left[V_{N}-V_{K}\right] & =h_{N}(\cdot)-g_{K}(\cdot)+p_{U, E}\left[V_{K}-V_{N}\right]-\alpha s_{K}\left[V_{N}-V_{K}\right]+p_{E, N}\left[V_{E}-V_{N}\right]
\end{aligned}
$$


implying

$$
\begin{aligned}
\rho\left[V_{E}-V_{U}\right] & =h_{E}(\cdot)-g_{U}(\cdot)+p_{U, E}\left[V_{U}-V_{E}\right]-\alpha s_{U}\left[V_{E}-V_{U}\right]-p_{K, U}\left[V_{K}-V_{E}+V_{E}-V_{U}\right] \\
\rho\left[V_{N}-V_{K}\right] & =h_{N}(\cdot)-g_{K}(\cdot)+p_{U, E}\left[V_{K}-V_{N}\right]-\alpha s_{K}\left[V_{N}-V_{K}\right]+p_{E, N}\left[V_{E}-V_{K}+V_{K}-V_{N}\right]
\end{aligned}
$$

and thus

$$
\begin{aligned}
{\left[V_{E}-V_{U}\right] } & =\frac{h_{E}(\cdot)-g_{U}(\cdot)-p_{K, U}\left[V_{K}-V_{E}\right]}{\rho+p_{U, E}+\alpha s_{U}+p_{K, U}} \\
{\left[V_{N}-V_{K}\right] } & =\frac{h_{N}(\cdot)-g_{K}(\cdot)-p_{E, N}\left[V_{K}-V_{E}\right]}{\rho+p_{U, E}+\alpha s_{K}+p_{E, N}} .
\end{aligned}
$$

Using that

$$
\rho\left[V_{K}-V_{E}\right]=g_{K}(\cdot)-h_{E}(\cdot)+\alpha s_{K}\left[V_{N}-V_{K}\right]-p_{U, E}\left[V_{U}-V_{E}\right]
$$

(11) and (12) implies

$$
\begin{aligned}
{\left[\rho+p_{U, E}+\alpha s_{U}+p_{K, U}\right]\left[V_{E}-V_{U}\right]=} & h_{E}(\cdot)-g_{U}(\cdot)-\frac{p_{K, U}}{\rho}\left[g_{K}(\cdot)-h_{E}(\cdot)\right. \\
& \left.+\alpha s_{K}\left[V_{N}-V_{K}\right]-p_{U, E}\left[V_{U}-V_{E}\right]\right] \\
{\left[\rho+p_{U, E}+\alpha s_{K}+p_{E, N}\right]\left[V_{N}-V_{K}\right]=} & h_{N}(\cdot)-g_{K}(\cdot)-\frac{p_{E, N}}{\rho}\left[g_{K}(\cdot)-h_{E}(\cdot)\right. \\
& \left.+\alpha s_{K}\left[V_{N}-V_{K}\right]-p_{U, E}\left[V_{U}-V_{E}\right]\right]
\end{aligned}
$$

which in turn can be written

$$
\begin{aligned}
{\left[\rho+p_{U, E}+\alpha s_{U}+p_{K, U}+\frac{p_{K, U}}{\rho} p_{U, E}\right]\left[V_{E}-V_{U}\right]=} & h_{E}(\cdot)-g_{U}(\cdot)+\frac{p_{K, U}}{\rho}\left[h_{E}(\cdot)-g_{K}(\cdot)\right. \\
& \left.-\alpha s_{K}\left[V_{N}-V_{K}\right]\right] \\
{\left[\rho+p_{U, E}+\alpha s_{K}+p_{E, N}+\frac{p_{E, N}}{\rho} \alpha s_{K}\right]\left[V_{N}-V_{K}\right]=} & h_{N}(\cdot)-g_{K}(\cdot)+\frac{p_{E, N}}{\rho}\left[h_{E}(\cdot)-g_{K}(\cdot)\right. \\
& \left.-p_{U, E}\left[V_{E}-V_{U}\right]\right] .
\end{aligned}
$$

Totally differentiating yields

$$
\begin{aligned}
{\left[V_{E}-V_{U}\right]\left(1+\frac{p_{U, E}}{\rho}\right) d p_{K, U}+A_{1} d\left[V_{E}-V_{U}\right] } & =\frac{1}{\rho} A_{2} d p_{K, U}-\frac{p_{K, U}}{\rho} \alpha s_{K} d\left[V_{N}-V_{K}\right] \\
{\left[V_{N}-V_{K}\right]\left[1+\frac{1}{\rho} \alpha s_{K}\right] d p_{E, N}+B_{1} d\left[V_{N}-V_{K}\right] } & =\frac{1}{\rho} B_{2} d p_{E, N}-\frac{p_{E, N}}{\rho} p_{U, E} d\left[V_{E}-V_{U}\right]
\end{aligned}
$$

where

$$
\begin{aligned}
A_{1} & \equiv\left[\rho+p_{U, E}+\alpha s_{U}+p_{K, U}+\frac{p_{K, U}}{\rho} p_{U, E}\right]>0 \\
A_{2} & \equiv\left[h_{E}(\cdot)-g_{K}(\cdot)-\alpha s_{K}\left[V_{N}-V_{K}\right]\right] \lesseqgtr 0 \\
B_{1} & \equiv \rho+p_{U, E}+\alpha s_{K}+p_{E, N}+\frac{p_{E, N}}{\rho} \alpha s_{K}>0 \\
B_{2} & \equiv h_{E}(\cdot)-g_{K}(\cdot)-p_{U, E}\left[V_{E}-V_{U}\right] \lesseqgtr 0 .
\end{aligned}
$$


Hence,

$$
\begin{aligned}
A_{1} d\left[V_{E}-V_{U}\right]= & {\left[\frac{1}{\rho} A_{2}-\left[V_{E}-V_{U}\right]\left(1+\frac{p_{U, E}}{\rho}\right)\right] d p_{K, U} } \\
& -\frac{p_{K, U}}{\rho} \alpha s_{K} d\left[V_{N}-V_{K}\right] \\
B_{1} d\left[V_{N}-V_{K}\right]= & {\left[\frac{1}{\rho} B_{2}-\left[V_{N}-V_{K}\right]\left[1+\frac{1}{\rho} \alpha s_{K}\right]\right] d p_{E, N} } \\
& -\frac{p_{E, N}}{\rho} p_{U, E} d\left[V_{E}-V_{U}\right] .
\end{aligned}
$$

Before proceeding we prove that

$$
\frac{1}{\rho} B_{2}-\left[V_{N}-V_{K}\right]\left[1+\frac{1}{\rho} \alpha s_{K}\right]>0
$$

or

$$
h_{E}(\cdot)-g_{K}(\cdot)-p_{U, E}\left[V_{E}-V_{U}\right]>\left[V_{N}-V_{K}\right]\left[\rho+\alpha s_{K}\right] .
$$

We have from the value functions that

$$
\left[\rho+\alpha s_{K}\right]\left[V_{N}-V_{K}\right]=h_{N}(\cdot)-g_{K}(\cdot)+p_{U, E}\left[V_{K}-V_{N}\right]+p_{E, N}\left[V_{E}-V_{N}\right]
$$

and hence, the inequality can be rewritten

$$
h_{E}(\cdot)-h_{N}(\cdot)-p_{U, E}\left[V_{E}-V_{U}\right]>p_{U, E}\left[V_{K}-V_{N}\right]+p_{E, N}\left[V_{E}-V_{N}\right] .
$$

Using that

$$
\rho\left[V_{E}-V_{N}\right]=h_{E}(\cdot)-h_{N}(\cdot)+p_{U, E}\left[V_{U}-V_{E}\right]-p_{U, E}\left[V_{K}-V_{N}\right]-p_{E, N}\left[V_{E}-V_{N}\right]
$$

we have that the inequality reduces to

$$
\rho\left[V_{E}-V_{N}\right]>0
$$

which is fulfilled.

We also prove that $\frac{1}{\rho} A_{2}-\left[V_{E}-V_{U}\right]\left(1+\frac{p_{U, E}}{\rho}\right)>0$ or

$$
h_{E}(\cdot)-g_{K}(\cdot)-\alpha s_{K}\left[V_{N}-V_{K}\right]>\left[V_{E}-V_{U}\right]\left(\rho+p_{U, E}\right) .
$$

We have from the value functions that

$$
\left(\rho+p_{U, E}\right)\left[V_{E}-V_{U}\right]=h_{E}(\cdot)-g_{U}(\cdot)-\alpha s_{U}\left[V_{E}-V_{U}\right]-p_{K, U}\left[V_{K}-V_{U}\right]
$$


and hence, the inequality can be written

$$
g_{U}(\cdot)-g_{K}(\cdot)>\alpha s_{K}\left[V_{N}-V_{K}\right]-\alpha s_{U}\left[V_{E}-V_{U}\right]-p_{K, U}\left[V_{K}-V_{U}\right] .
$$

Using that

$$
\rho\left[V_{U}-V_{K}\right]=g_{U}(\cdot)-g_{K}(\cdot)+\alpha s_{U}\left[V_{E}-V_{U}\right]+p_{K, U}\left[V_{K}-V_{U}\right]-\alpha s_{K}\left[V_{N}-V_{K}\right]
$$

the inequality reduces to

$$
\rho\left[V_{U}-V_{K}\right]>0
$$

which is fulfilled.

Finally, also note that

$$
B_{1}-\frac{p_{E, N}}{\rho} p_{U, E} \frac{p_{K, U}}{\rho} \frac{\alpha s_{K}}{A_{1}}=\frac{1}{A_{1}}\left[A_{1} B_{1}-\frac{p_{E, N}}{\rho} p_{U, E} \frac{p_{K, U}}{\rho} \alpha s_{K}\right]>0 .
$$

Returning to (13) and (14) we have for $d p_{K, U}=0$ that

$$
\begin{aligned}
& A_{1} d\left[V_{E}-V_{U}\right]=-\frac{p_{K, U}}{\rho} \alpha s_{K} d\left[V_{N}-V_{K}\right] \\
& {\left[B_{1}-\frac{p_{E, N}}{\rho} p_{U, E} \frac{p_{K, U}}{\rho} \frac{\alpha s_{K}}{A_{1}}\right] d\left[V_{N}-V_{K}\right]=\left[\frac{1}{\rho} B_{2}-\left[V_{N}-V_{K}\right]\left[1+\frac{1}{\rho} \alpha s_{K}\right]\right] d p_{E, N}} \\
& d\left[V_{N}-V_{K}\right]=\frac{\left[\frac{1}{\rho} B_{2}-\left[V_{N}-V_{K}\right]\left[1+\frac{1}{\rho} \alpha s_{K}\right]\right]}{\left[B_{1}-\frac{p_{E, N}}{\rho} p_{U, E} \frac{p_{K, U}}{\rho} \frac{\alpha s_{K}}{A_{1}}\right]} d p_{E, N} \\
& d\left[V_{E}-V_{U}\right]=-\frac{1}{A_{1}} \frac{p_{K, U}}{\rho} \alpha s_{K} \frac{\left[\frac{1}{\rho} B_{2}-\left[V_{N}-V_{K}\right]\left[1+\frac{1}{\rho} \alpha s_{K}\right]\right]}{\left[B_{1}-\frac{p_{E, N}}{\rho} p_{U, E} \frac{p_{K, U}}{\rho} \frac{\alpha s_{K}}{A_{1}}\right]} d p_{E, N} \\
& \frac{d\left[V_{N}-V_{K}\right]}{d\left[V_{E}-V_{U}\right]}=\left[-\frac{1}{A_{1}} \frac{p_{K, U}}{\rho} \alpha s_{K}\right]^{-1} \\
& \frac{d\left[V_{N}-V_{K}\right]}{d p_{E, N}}=\frac{\left[\frac{1}{\rho} B_{2}-\left[V_{N}-V_{K}\right]\left[1+\frac{1}{\rho} \alpha s_{K}\right]\right]}{\left[B_{1}-\frac{p_{E, N}}{\rho} p_{U, E} \frac{p_{K, U}}{\rho} \frac{\alpha s_{K}}{A_{1}}\right]} \\
& \frac{d\left[V_{E}-V_{U}\right]}{d p_{E, N}}=-\frac{1}{A_{1}} \frac{p_{K, U}}{\rho} \alpha s_{K} \frac{\left[\frac{1}{\rho} B_{2}-\left[V_{N}-V_{K}\right]\left[1+\frac{1}{\rho} \alpha s_{K}\right]\right]}{\left[B_{1}-\frac{p_{E, N}}{\rho} p_{U, E} \frac{p_{K, U}}{\rho} \frac{\alpha s_{K}}{A_{1}}\right]} .
\end{aligned}
$$


and for $d p_{E, N}=0$ that

$$
\begin{aligned}
& B_{1} d\left[V_{N}-V_{K}\right]=-\frac{p_{E, N}}{\rho} p_{U, E} d\left[V_{E}-V_{U}\right] \\
& {\left[A_{1}-\frac{p_{E, N}}{\rho} p_{U, E} \frac{p_{K, U}}{\rho} \frac{\alpha s_{K}}{B_{1}}\right] d\left[V_{E}-V_{U}\right]=\left[\frac{1}{\rho} A_{2}-\left[V_{E}-V_{U}\right]\left(1+\frac{p_{U, E}}{\rho}\right)\right] d p_{K, U}} \\
& d\left[V_{E}-V_{U}\right]=\frac{\left[\frac{1}{\rho} A_{2}-\left[V_{E}-V_{U}\right]\left(1+\frac{p_{U, E}}{\rho}\right)\right]}{\left[A_{1}-\frac{p_{E, N}}{\rho} p_{U, E} \frac{p_{K, U}}{\rho} \frac{\alpha s_{K}}{B_{1}}\right]} d p_{K, U} \\
& d\left[V_{N}-V_{K}\right]=\frac{-1}{B_{1}} \frac{p_{E, N}}{\rho} p_{U, E} \frac{\left[\frac{1}{\rho} A_{2}-\left[V_{E}-V_{U}\right]\left(1+\frac{p_{U, E}}{\rho}\right)\right]}{\left[A_{1}-\frac{p_{E, N}}{\rho} p_{U, E} \frac{p_{K, U}}{\rho} \frac{\alpha s_{K}}{B_{1}}\right]} d p_{K, U} \\
& \frac{d\left[V_{N}-V_{K}\right]}{d\left[V_{E}-V_{U}\right]}=\frac{-1}{B_{1}} \frac{p_{E, N}}{\rho} p_{U, E} \\
& \frac{d\left[V_{E}-V_{U}\right]}{d p_{K, U}}=\frac{\left[\frac{1}{\rho} A_{2}-\left[V_{E}-V_{U}\right]\left(1+\frac{p_{U, E}}{\rho}\right)\right]}{\left[A_{1}-\frac{p_{E, N}}{\rho} p_{U, E} \frac{p_{K, U}}{\rho} \frac{\alpha s_{K}}{B_{1}}\right]} \\
& \frac{d\left[V_{N}-V_{K}\right]}{d p_{K, U}}=\frac{-1}{B_{1}} \frac{p_{E, N}}{\rho} p_{U, E} \frac{\left[\frac{1}{\rho} A_{2}-\left[V_{E}-V_{U}\right]\left(1+\frac{p_{U, E}}{\rho}\right)\right]}{\left[A_{1}-\frac{p_{E, N}}{\rho} p_{U, E} \frac{p_{K, U}}{\rho} \frac{\alpha s_{K}}{B_{1}}\right]} .
\end{aligned}
$$

Hence, we have established the following signs

\begin{tabular}{c|cc} 
& $d\left[V_{N}-V_{K}\right]$ & $d\left[V_{E}-V_{U}\right]$ \\
\hline$d p_{E, N}$ & $>0$ & $<0$ \\
$d p_{K, U}$ & $<0$ & $>0$
\end{tabular}.

This implies that

$$
\begin{aligned}
\frac{d s_{U}}{d p_{K, U}}>0 ; \frac{d s_{K}}{d p_{K, U}}<0 \\
\frac{d s_{U}}{d p_{E, N}}<0 ; \frac{d s_{K}}{d p_{E, N}}>0 .
\end{aligned}
$$

\section{Marginal rates of substitution}

Consider next the marginal rates of return, i.e., combinations of $p_{K, U}$ and $p_{E, N}$ leaving $V_{E}-V_{U}$ and thus search $s_{U}$ unchanged (and similarly for $s_{K}$ ). Using

$$
\begin{aligned}
A_{1} d\left[V_{E}-V_{U}\right] & =\left[\frac{1}{\rho} A_{2}-\left[V_{E}-V_{U}\right]\left(1+\frac{p_{U, E}}{\rho}\right)\right] d p_{K, U}-\frac{p_{K, U}}{\rho} \alpha s_{K} d\left[V_{N}-V_{K}\right] \\
B_{1} d\left[V_{N}-V_{K}\right] & =\left[\frac{1}{\rho} B_{2}-\left[V_{N}-V_{K}\right]\left[1+\frac{1}{\rho} \alpha s_{K}\right]\right] d p_{E, N}-\frac{p_{E, N}}{\rho} p_{U, E} d\left[V_{E}-V_{U}\right]
\end{aligned}
$$


and imposing $d\left[V_{E}-V_{U}\right]=0$, we obtain

$0=\left[\frac{1}{\rho} A_{2}-\left[V_{E}-V_{U}\right]\left(1+\frac{p_{U, E}}{\rho}\right)\right] d p_{K, U}-\frac{p_{K, U}}{\rho} \frac{\alpha s_{K}}{B_{1}}\left[\frac{1}{\rho} B_{2}-\left[V_{N}-V_{K}\right]\left[1+\frac{1}{\rho} \alpha s_{K}\right]\right] d p_{E, N}$

and hence,

$$
\left.\frac{d p_{K, U}}{d p_{E, N}}\right|_{\left[V_{E}-V_{U}\right]=\mathrm{constant}}=\frac{\frac{p_{K, U}}{\rho} \frac{\alpha s_{K}}{B_{1}}\left[\frac{1}{\rho} B_{2}-\left[V_{N}-V_{K}\right]\left[1+\frac{1}{\rho} \alpha s_{K}\right]\right]}{\left[\frac{1}{\rho} A_{2}-\left[V_{E}-V_{U}\right]\left(1+\frac{p_{U, E}}{\rho}\right)\right]}>0 .
$$

Similarly, for $s_{K}$ where we have that $d\left[V_{N}-V_{K}\right]=0$ implies

$0=\left[\frac{1}{\rho} B_{2}-\left[V_{N}-V_{K}\right]\left[1+\frac{1}{\rho} \alpha s_{K}\right]\right] d p_{E, N}-\frac{p_{E, N}}{\rho} \frac{p_{U, E}}{A_{1}}\left[\frac{1}{\rho} A_{2}-\left[V_{E}-V_{U}\right]\left(1+\frac{p_{U, E}}{\rho}\right)\right] d p_{K, U}$

and hence,

$$
\left.\frac{d p_{E, N}}{d p_{K, U}}\right|_{\left[V_{N}-V_{K}\right]=\mathrm{const}}=\frac{\frac{p_{E, N}}{\rho} \frac{p_{U, E}}{A_{1}}\left[\frac{1}{\rho} A_{2}-\left[V_{E}-V_{U}\right]\left(1+\frac{p_{U, E}}{\rho}\right)\right]}{\left[\frac{1}{\rho} B_{2}-\left[V_{N}-V_{K}\right]\left[1+\frac{1}{\rho} \alpha s_{K}\right]\right]}>0 .
$$

Note that (recall that $A_{1}>0$ and $B_{1}>0$ )

$$
\left.\left.\frac{d p_{K, U}}{d p_{E, N}}\right|_{\left[V_{E}-V_{U}\right]=\text { constant }} \frac{d p_{E, N}}{d p_{K, U}}\right|_{\left[V_{N}-V_{K}\right]=\mathrm{const}}=\frac{p_{K, U}}{\rho} \frac{\alpha s_{K}}{B_{1}} \frac{p_{E, N}}{\rho} \frac{p_{U, E}}{A_{1}} \lesseqgtr 1 .
$$

From the envelope theorem we know that the utility effect of a given policy change is given by the direct utility effects (all indirect effects via behavior wash out via first order conditions).

\begin{tabular}{c|cc} 
& $d\left[V_{N}-V_{K}\right]$ & $d\left[V_{E}-V_{U}\right]$ \\
\hline$d p_{E, N}$ & $>0$ & $<0$ \\
$d p_{K, U}$ & $<0$ & $>0$
\end{tabular}

and

$$
\begin{aligned}
\rho V_{E} & =h(w[1-\tau]+\Pi, 1-l)+p_{U, E}\left[V_{U}-V_{E}\right] \\
\rho V_{N} & =h(w[1-\tau]+\Pi, 1-l)+p_{U, E}\left[V_{K}-V_{N}\right]+p_{E, N}\left[V_{E}-V_{N}\right] \\
\rho V_{U} & =g\left(b_{U}+\Pi, 1-s_{U}\right)+\alpha s_{U}\left[V_{E}-V_{U}\right]+p_{K, U}\left[V_{K}-V_{U}\right] \\
\rho V_{K} & =g\left(b_{K}+\Pi, 1-s_{K}\right)+\alpha s_{K}\left[V_{N}-V_{K}\right]
\end{aligned}
$$

we thus have: i) an increase in $p_{K, U}$ leads to a decrease in $V_{E}$ and a decrease in $V_{K}$, while there is an ambiguous effect on $V_{N}$ and $V_{U}$, ii) an increase in $p_{E, N}$ leads to an increase in $V_{E}$ and an increase in $V_{K}$, while there is an ambiguous effect on $V_{N}$ and $V_{U}$. 


\section{Iso-gross unemployment loci}

Note first that we have

$$
u=\frac{p_{E, N}}{p_{K, U}} n
$$

implying

$$
k=\frac{p_{U, E}+p_{E, N}}{\alpha s_{K}} n=\frac{p_{U, E}+p_{E, N}}{\alpha s_{K}} \frac{p_{K, U}}{p_{E, N}} u
$$

and

$$
u=\frac{p_{U, E}}{\alpha s_{U}+p_{K, U}+p_{U, E}+p_{U, E} \frac{p_{U, E}+p_{E, N}}{\alpha s_{K}} \frac{p_{K, U}}{p_{E, N}}+p_{U, E} \frac{p_{K, U}}{p_{E, N}}}
$$

$e=1-u-n-k$

$$
\begin{aligned}
= & 1-\left(1-\frac{p_{K, U}}{p_{E, N}}-\frac{p_{U, E}+p_{E, N}}{\alpha s_{K}} \frac{p_{K, U}}{p_{E, N}}\right) \frac{p_{U, E}}{\alpha s_{U}+p_{K, U}+p_{U, E}+p_{U, E} \frac{p_{U, E}+p_{E, N}}{\alpha s_{K}} \frac{p_{K, U}}{p_{E, N}}+p_{U, E} \frac{p_{K, U}}{p_{E, N}}} \\
= & 1-\left(\frac{p_{E, N} \alpha s_{K}-\left(\alpha s_{K}+p_{U, E}+p_{E, N}\right) p_{K, U}}{\alpha s_{K} p_{E, N}}\right) \\
& \times \frac{p_{U, E}}{\alpha s_{U}+p_{K, U}+p_{U, E}+p_{U, E} \frac{p_{U, E}+p_{E, N}}{\alpha s_{K}} \frac{p_{K, U}}{p_{E, N}}+p_{U, E} \frac{p_{K, U}}{p_{E, N}}} .
\end{aligned}
$$

It follows that gross unemployment $(u+k)$ is given as

$$
\begin{aligned}
u+k & =u\left[1+\frac{p_{U, E}+p_{E, N}}{\alpha s_{K}} \frac{p_{K, U}}{p_{E, N}}\right] \\
& =\frac{p_{U, E}\left[1+\frac{p_{U, E}+p_{E, N}}{\alpha s_{K}} \frac{p_{K, U}}{p_{E, N}}\right]}{\alpha s_{U}+p_{K, U}+p_{U, E}+p_{U, E} \frac{p_{U, E}+p_{E, N}}{\alpha s_{K}} \frac{p_{K, U}}{p_{E, N}}+p_{U, E} \frac{p_{K, U}}{p_{E, N}}}<1
\end{aligned}
$$

or

$$
\begin{aligned}
\frac{1}{u+k}= & 1+\frac{\alpha s_{U}+p_{K, U}+p_{U, E} \frac{p_{K, U}}{p_{E, N}}}{p_{U, E}+\frac{p_{U, E}+p_{E, N}}{\alpha s_{K}} \frac{p_{K, U}}{p_{E, N}} p_{U, E}}>1 \\
= & 1+\frac{\alpha s_{U}+p_{K, U}+\frac{p_{U, E}}{p_{E, N}} p_{K, U}}{p_{U, E}+\frac{\frac{p_{U, E}}{p_{E, N}}+1}{\alpha s_{K}} p_{K, U} p_{U, E}}
\end{aligned}
$$

Where

$$
\begin{aligned}
\frac{\partial}{\partial s_{U}}\left(\frac{1}{u+k}\right) & =\frac{\alpha}{p_{U, E}+\frac{\frac{p_{U, E}}{p_{E, N}}+1}{\alpha s_{K}} p_{K, U} p_{U, E}}>0 \\
\frac{\partial}{\partial s_{K}}\left(\frac{1}{u+k}\right) & =\frac{\alpha s_{U}+p_{K, U}+\frac{p_{U, E}}{p_{E, N}} p_{K, U}}{\left[p_{U, E}+\frac{\frac{p_{U, E}}{p_{E, N}}+1}{\alpha s_{K}} p_{K, U} p_{U, E}\right]^{2}}\left[\alpha \frac{\frac{p_{U, E}}{p_{E, N}}+1}{\left[\alpha s_{K}\right]^{2}} p_{K, U} p_{U, E}\right]>0
\end{aligned}
$$




$$
\begin{aligned}
& p_{K, U}\left[p_{U, E}+\frac{\frac{p_{U, E}}{p_{E}, N}+1}{\alpha s_{K}} p_{K, U} p_{U, E}\right] \\
& \frac{\partial}{\partial p_{E N}}\left(\frac{1}{u+k}\right)=\frac{-\left[\alpha s_{U}+p_{K, U}+\frac{p_{U, E}}{p_{E, N}} p_{K, U}\right]\left[\frac{p_{K, U} p_{U, E}}{\alpha s_{K}}\right]}{\left[p_{U, E}+\frac{\frac{p_{U, E}}{p_{E, N}}+1}{\alpha s_{K}} p_{K, U} p_{U, E}\right]^{2}}\left(-\frac{p_{U, E}}{p_{E, N}^{2}}\right) \\
& =\frac{p_{K, U}\left[p_{U, E}+\frac{1}{\alpha s_{K}} p_{K, U} p_{U, E}\right]-\left[\alpha s_{U}+p_{K, U}\right]\left[\frac{p_{K, U} p_{U, E}}{\alpha s_{K}}\right]}{\left[p_{U, E}+\frac{\frac{p_{U, E}}{p_{E, N}}+1}{\alpha s_{K}} p_{K, U} p_{U, E}\right]^{2}}\left(-\frac{p_{U, E}}{p_{E, N}^{2}}\right) \\
& =\frac{p_{K, U} p_{U, E}\left[1-\frac{s_{U}}{s_{K}}\right]}{\left[p_{U, E}+\frac{\frac{p_{U, E}}{p_{E, N}}+1}{\alpha s_{K}} p_{K, U} p_{U, E}\right]^{2}}\left(-\frac{p_{U, E}}{p_{E, N}^{2}}\right) \lesseqgtr 0 \text { for } \frac{s_{U}}{s_{K}} \lesseqgtr 1 \\
& \frac{\partial}{\partial p_{K U}}\left(\frac{1}{u+k}\right)=\frac{-\left[\alpha s_{U}+p_{K, U}+\frac{p_{U, E}}{p_{E, N}}\right]\left[p_{U, E}+\frac{\frac{p_{U, E}}{p_{E, N}}+1}{\alpha s_{K}} p_{K, U}\right]\left[\frac{\frac{p_{U, E}}{p_{E, N}}+1}{\alpha s_{K}} p_{U, E}\right]}{\left[p_{U, E}+\frac{\frac{p_{U, E}}{p_{E, N}}+1}{\alpha s_{K}} p_{K, U} p_{U, E}\right]^{2}} \\
& =\frac{\left[1+\frac{p_{U, E}}{p_{E, N}}\right]\left[p_{U, E}+\frac{p_{K, U} p_{U, E}}{\alpha s_{K}}\right]-\left[\alpha s_{U}+p_{K, U}\right]\left[\frac{\frac{p_{U, E}}{p_{E, N}}+1}{\alpha s_{K}} p_{U, E}\right]}{\left[p_{U, E}+\frac{\frac{p_{U, E}}{p_{E, N}}+1}{\alpha s_{K}} p_{K, U} p_{U, E}\right]^{2}} \\
& =\frac{p_{U, E}-\alpha s_{U} \frac{1}{\alpha s_{K}} p_{U, E}}{\left[p_{U, E}+\frac{\frac{p_{U, E}}{p_{E, N}}+1}{\alpha s_{K}} p_{K, U} p_{U, E}\right]^{2}}\left[1+\frac{p_{U, E}}{p_{E, N}}\right]<0 \\
& =\frac{p_{U, E}\left(1-\frac{s_{U}}{s_{K}}\right)\left[1+\frac{p_{U, E}}{p_{E, N}}\right]}{\left[p_{U, E}+\frac{\frac{p_{U, E}}{p_{E, N}}+1}{\alpha s_{K}} p_{K, U} p_{U, E}\right]^{2}} \lesseqgtr 0 \text { for } \frac{s_{U}}{s_{K}} \gtreqless 1 .
\end{aligned}
$$

Hence, the gross unemployment can be written in implicit form as

$$
u+k=F\left(s_{U}\left(p_{K, U}, p_{E, N}\right), s_{K}\left(p_{K, U}, p_{E, N}\right), p_{K, U}, p_{E, N}\right)
$$

Note that

$$
\operatorname{sign} \frac{\partial F(\cdot)}{\partial z}=-\operatorname{sign} \frac{\partial}{\partial z}\left(\frac{1}{u+k}\right)
$$


It follows that

$$
\begin{aligned}
\frac{\partial F(\cdot)}{\partial s_{U}} & <0 ; \frac{\partial F(\cdot)}{\partial s_{K}}<0 \\
\operatorname{sign}\left(\frac{\partial F(\cdot)}{\partial p_{K, U}}\right) & =\operatorname{sign}\left(s_{U}-s_{K}\right) \\
\operatorname{sign}\left(\frac{\partial F(\cdot)}{\partial p_{E, N}}\right) & =-\operatorname{sign}\left(\frac{\partial F(\cdot)}{\partial p_{K, U}}\right) .
\end{aligned}
$$

We have that

$$
\begin{aligned}
d(u+k)= & \frac{\partial F(\cdot)}{\partial s_{U}} d s_{U}+\frac{\partial F(\cdot)}{\partial s_{K}} d s_{K}+\frac{\partial F(\cdot)}{\partial p_{K, U}} d p_{K, U}+\frac{\partial F(\cdot)}{\partial p_{E, N}} d p_{E, N} \\
= & \frac{\partial F(\cdot)}{\partial s_{U}}\left[\frac{\partial s_{U}}{\partial p_{K, U}} d p_{K, U}+\frac{\partial s_{U}}{\partial p_{E, N}} d p_{E, N}\right]+\frac{\partial F(\cdot)}{\partial s_{K}}\left[\frac{\partial s_{K}}{\partial p_{K, U}} d p_{K, U}+\frac{\partial s_{K}}{\partial p_{E, N}} d p_{E, N}\right] \\
& +\frac{\partial F(\cdot)}{\partial p_{K, U}} d p_{K, U}+\frac{\partial F(\cdot)}{\partial p_{E, N}} d p_{E, N} .
\end{aligned}
$$

For $d(u+k)=0$ we have

$$
\begin{aligned}
& -\left[\frac{\partial F(\cdot)}{\partial p_{E, N}}+\frac{\partial s_{U}}{\partial p_{E, N}} \frac{\partial F(\cdot)}{\partial s_{U}}+\frac{\partial s_{K}}{\partial p_{E, N}} \frac{\partial F(\cdot)}{\partial s_{K}}\right] d p_{E, N} \\
= & {\left[\frac{\partial F(\cdot)}{\partial p_{K, U}}+\frac{\partial s_{K}}{\partial p_{K, U}} \frac{\partial F(\cdot)}{\partial s_{K}}+\frac{\partial s_{U}}{\partial p_{K, U}} \frac{\partial F(\cdot)}{\partial s_{U}}\right] d p_{K, U} } \\
\frac{d p_{E, N}}{d p_{K, U}}= & -\frac{\frac{\partial F(\cdot)}{\partial p_{K, U}}+\frac{\partial s_{K}}{\partial p_{K, U}} \frac{\partial F(\cdot)}{\partial s_{K}}+\frac{\partial s_{U}}{\partial p_{K, U}} \frac{\partial F(\cdot)}{\partial s_{U}}}{\frac{\partial s_{U}}{\partial p_{E, N}}+\frac{\partial F(\cdot)}{\partial p_{E, N}}+\frac{\partial s_{K}}{\partial s_{U}} \frac{\partial F(\cdot)}{\partial s_{K}}}
\end{aligned}
$$

Notice that

$$
\begin{aligned}
\frac{d s_{U}}{d p_{K, U}}>0 ; \frac{d s_{K}}{d p_{K, U}}<0 \\
\frac{d s_{U}}{d p_{E, N}}<0 ; \frac{d s_{K}}{d p_{E, N}}>0 .
\end{aligned}
$$

Hence, the numerator and denominator of (15) are in general ambiguously signed. 